\title{
Renewable Energy Driven by Le Chatelier's Principle, Enzyme Function, and Non-Additive Contributions to Ion Fluctuations: A Hypothesis in Biomechanical and Nanotechnology Energy Theory
}

\author{
Sergio Manzetti \\ Nanotoxicology Unit, Western Norway Research Institute, Fossahaugane Campus, 6851 Sogndal, Norway \\ Correspondence should be addressed to Sergio Manzetti, sergio.manzetti@vestforsk.no
}

Received 11 November 2010; Accepted 17 January 2011

Academic Editor: John A. Capobianco

Copyright ( $) 2011$ Sergio Manzetti. This is an open access article distributed under the Creative Commons Attribution License, which permits unrestricted use, distribution, and reproduction in any medium, provided the original work is properly cited.

\begin{abstract}
The search for green energy sources has populated the research arena with significant emphasis on green electronics, green fuels, and green batteries that reduce waste, emissions, and environmental toxicity. Simultaneously, nanotechnology has developed substantially in the recent years and the emerging area of nanoenergetics has shown impressive discoveries that can aid in the search for alternative and green energies. The use of exotic materials in these fields and even enzymes has led scientists to be able to cross-link biomolecules and nanotechnology circuits, which can be important points in the search of novel energy searches. This paper discusses a biochemical energy-generating unit driven by ion fluctuations and spontaneous enzyme conformational changes. The paper lays also the theoretical thermodynamical foundation of the nanoenergy unit and to exploit the principle of nonadditivity and equilibrium as main forces in driving an energy-generating reaction.
\end{abstract}

\section{Introduction}

The energy crisis has escalated within the last 2 decades and the search for greener energies has increased significantly [14]. The search for green energy and technologies encompasses novel battery technologies, alternative fuels, biofuels and other technologies that generate no or low waste products. Simultaneously, nanotechnology has been gradually introduced in the field of energy search [5-7]. Nanotechnology has developed at a fast pace within the last 10 years where the use nanomaterials such as carbon nanotubes, nanowires, quantum dots and nanocomposites with particular properties are increasingly applied in the industry. The use of nanoelectronical circuits and nanoenergetics is increasingly emerging, particularly in respect with green electronics. In this field smaller and smaller units are constructed to produce functionalities as rotation, molecular recognition, superconductivity and other nanosized interactions.

The use of enzymes in exploration of energy sources in nanotechnology have been recently explored [8-10] however in a limited degree with relevance to the field of green energy sources. Enzymes are biomolecular units that serve the cell's function in a multitude of ways. The biomechanical and biochemical aspects of enzymes allow reactions to occur several times faster than in regular chemistry. In order to use more of this knowledge, enzymes are in this paper explored as possible components in nanosized units that can serve in the search in green energy sources. This paper aims to explore aspects of cellular biology which can be inspiring to heuristic and theoretical nanotechnology in green energy theory. This study also presents a structure of a nanocell with its theoretical foundation and thermodynamical considerations.

\section{Generation of Energy Using Enzymes as Working Units}

The aspects explored here centers particularly on generating electricity from nanotechnology circuits including biomolecular "machines". As an example from nature, 
the electron transport chain is known to generate a potential to convert ADP into ATP by building a difference in concentration of $\mathrm{H}^{+}$-ions between one side of a membrane to the other. In order to allow such energy conditions to arise within the mitochondria, the metabolism of glucose allows the proper proton transfer to the intermembrane space. Given that the mitochondria are the cell's energy source, their structure with an intermembrane space, multiple cell membranes and designated integrations of ATPsyntases, cytochromes and other electron transport factors have inspired the authors here to hypothesize the structure of a nanosized fuel cell with a similar architecture. Here discussed, the hypothesized system reminds very much of a battery/osmosis principle (see Figure 1) and is composed of multiple layers, aiming to generate electrostatic potentials to drive an energy-generating function in an inspired fashion to mitochondria. Knowing that enzymes accelerate chemical reactions thousand to hundred thousandfold, the enzymes' potential in recharging or simply generating electricity in nanoelectronic systems is hypothesized to surpass modern day batteries and energy sources if soundly constructed. The theory of incorporating enzymes in multiple energygenerating units in a novel fashion is explored here using a group of proteins called Voltage-gated ion-transfer proteins [11-14].

Voltage-gated ion-transfer proteins are biomolecular bodies that are integrated in the cell membrane where they form a regulated channel which generates an electric potential across the cell membrane [11-15]. Via this channel they facilitate the influx and efflux of ions across the membrane (potassium, sodium, and calcium ions for instance) in a highly sophisticated manner. The mechanism of action has been elucidated [15] and shows that the protein is stretched $15 \AA$ through the pore of the membrane facilitating the ion transfer from the cytosol to the extracellular matrix. These proteins have interestingly a sensor domain, which reacts to a specific voltage on the surface of the membrane, and functions as a nanobioswitch for the proteins' function. The function itself is spontaneous as folding in the presence of ions, and requires potassium ions at low or high concentrations, creating tail-current voltage-activation responses [15]. The depolarization of the potassium currents measured in the referred study were in the range of $0 \mathrm{mV}$ and $60 \mathrm{mV}$ based on the concentrations applied [15] and triggered ATP-free macromolecular motions that generated pumping of ions from one side of the membrane to the other.

\section{A Nano-Ion-Fuel Cell: A View of Using Enzymes to Generate Energy}

The nanoenergy unit is first described in this paragraph, and then considered thermodynamically in the next section. The pumping function of the ion pump is considered as the main motor in the nanobattery where the ion pumps are oriented in opposite directions (Figure 1). The number of enzymes is not discussed here, but rather the option of counterdirecting enzymes in this manner. The thought of counterdirected pumps is hypothesized to give the potential in exploiting non-additive effects (to be explained in the next paragraph) on the triggering of the sensor domains and in making the charges in-homogenous in between the layers (upon the fabrication process). The population of ions within the membrane spaces is expected to be in continuous fluctuation where the counterbalanced pumps work continuously attempting to reach equilibrium and the artificially applied ionic concentration differences work to satisfy Le Chatelier's principle (outlined in the next paragraph). The pumps are located within the membranes as previously achieved, however with a twist: the voltage-gated ion pumps have their sensors on the inner and outer side of the multiple membranes, triggering thereby pumping actions based on the differences of ion-concentration induced by the inverse pumps. It is furthermore expected that non-additive contributions between the compartments with differing ion concentrations drive ionic fluctuations that again activate the sensor domains inducing repeated pumping functions. Therefore, exploiting the voltage-sensors sensitivity, the iontransferring function is continuously triggered in attempt to reach equilibrium. Equilibrium is hypothesized to be outside a reachable horizon, if a suitable amount of enzymes in the opposite direction of the effect exerted by Le Chatelier's contributions is chosen. The pushing force of the enzyme (see next paragraph) is hypothetically compared to the opposite force exerted by the principle of Le Chatelier, but not measured, given that this is a heuristic theoretical paper.

The hypothetical condition of nonequilibrium, if the cell is properly engineered, can drive the system even more efficiently through the addition of several layers over each other, increasing the number of intramembrane spaces prefilled with ions and thereby giving more electrostatic potentials for the voltage sensors to switch on and off to. This increases also the degree of complexity, and thereby the participation of non-additive contributions (explained in the next chapter) that supply with variations in the ion fluctuations to an extent that the cell may function autonomously without the need of recharging.

The whole system becomes in other words more affected by the internal non-additive contributions and Le Chatelier's principle-induced fluctuations of ions: inducing the pumps to continuously be activated (through voltage-fluctuations). Le Chatelier's principle states that ions would flow naturally from a high concentration to a low concentration of ions through pores in a membrane. However, when these pores are active channels that pump accordingly to their sensor's commands, Le Chatelier's Principle will attempt to counteract the enzymes function, and vice versa.

The enzymes' attempts to equilibrate the ion concentrations is used as labor in generating the energy, however these functions need to be translated into actual energy.

\section{Application of Nanogenerated Energy as Nanomechanical Energy Based on Existing Technologies}

The potential of generating nanomechanical energy lies in generating electricity using a sensitive nanocomposite in 


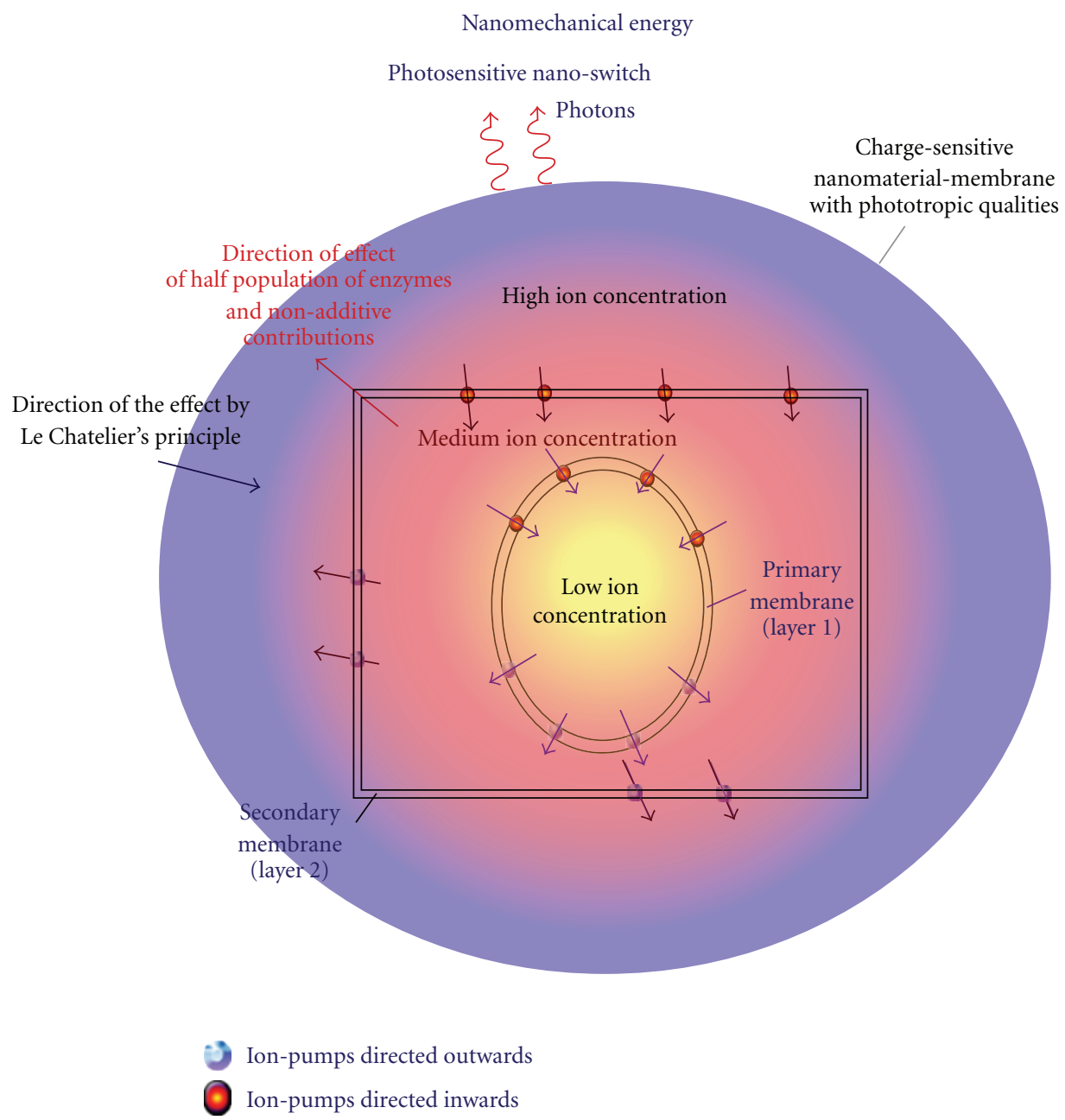

Figure 1: A hypothetical nanoenergy unit. The figure shows the potentially continuous exchange of ions between phases within a contained nanosystem. The system has two or more inner membranes, which act as phase-separators between phases of ion concentrations. The number of voltage pumps is chosen for illustrative purposes, and does not reflect (21). The effect of exchange of ions exploits the voltagedependent transmembrane ion-pumps innate need to equilibrate the ionic populations as proven [15]. Competing with each other, the two direction enzymes from the inner and outer membrane continuously pump ions in and out of the membranes generating a polarization effect on the surrounding nanoencapsulating material. Simultaneously Le Chatelier's principle exerts an additional force, which drives furthermore to stimulate the sensor domains' activation. The nanocomposite encapsulating the fuel cell bears phototrophic qualities emitting photons. The contribution of nonadditivity yields a system that theoretically does not reach equilibrium. If equilibrium is reached however, the application of magnets can be applied to recharge the unit by distributing the ions un-evenly again. The system, emitting photons to a photosensitive switch, transforms the energy into nanomechanical energy.

the containing membrane (see Figure 1), which carries nanoenergetics qualities such as phototrophic or mechanochromic qualities [16]. These qualities generate light impulses or change surface qualities in a fashion that is translatable to an energy generator. Through exploiting these properties, the system needs to be contained by a solid membrane and where its superficial changes are influenced by the inside. Reacting to ion fluxes, the encompassing nanocomposite is able to emit photonic signals to a photosensitive switch. Photosensitive chemistries are investigated, and are useful in nanotechnology as colloides and nanocomposites [17]. This very transfer of energy from a photosensitive switch to a nanomechanical unit is organized through the rotation of nanotubes [18]. The nanotube itself can promote the rotation of "nanoturbines" or units that interchange electrons in a rotating motion which can be developed based on recent findings in nanoelectric circuits [19]. The idea of applying nanoturbines or rotating units is a crucial aspect of nanotechnology research and particularly relevant in the generation of electricity from a nanoscale. The rotation of units at the nanoscopic level has been investigated by several groups [18-21] however in different nanosized environments. Nevertheless, rotation can be exploited to drive nanoturbines, which are technologically dependent on materials that are suitable to free their electrons during rotations to a surrounding nanocomposite, following the same system as in hydroelectricity turbines. From a nanotechnological point of view, this setting derives from the "nanoscale encarceration" 
principle as illustrated by Dr. Mann [22]. The channeling of electrons can be carried through the application of multiwalled nanotubes which may act as semiconductors [23] or via the application of nanowires [19, 24]. Ultimately the composition of the nanoturbines would have to be built with appropriate electric and magnetic nanomaterials with dipolar and paramagnetic properties [24-26]. This hypothetical circuit, where biomolecular force becomes electric energy is a fascinating suggestion which as hypothesized here could be proven as a valuable contribution in expanding the options of finding alternative energy sources.

\section{Thermodynamical Considerations for a Nano-Ion Cell.}

The entropy of the function of an enzyme is the true potential of a spontaneous reaction. A spontaneous reaction can be a conformational change, such that occurs in protein folding, and in the pumping motion of the voltage-gated ion pumps, where no substrates are required except ions. The spontaneity of these enzymes to react to their environment can be a key point to generate nanomechanical energy.

The entropy change of an enzyme during a process can be simplified as follows:

$$
\begin{aligned}
\Delta \mathrm{S}_{\text {enz }}= & \Delta \mathrm{S}_{\text {torsions }}+\Delta \mathrm{S}_{\text {rotations }}+\Delta \mathrm{S}_{\text {vibrations }}+\Delta \mathrm{S}_{\text {stretching }} \\
& +\Delta \mathrm{S}_{\mathrm{H} \text {-bond }}+\Delta \mathrm{S}_{\text {van der Waal }}+\Delta \mathrm{S}_{\text {Coloumb }}+\Delta \mathrm{S}_{\text {solvent }}+a,
\end{aligned}
$$

where $a$ encounters for other contributions to the total entropy of the enzyme, $\Delta \mathrm{S}_{\text {enz }}$.

In a relaxed state the entropy of the enzyme is given by

$$
\Delta \mathrm{S}_{\mathrm{enz}-\mathrm{relax}}
$$

and the entropy in an active state is given by

$$
\Delta \mathrm{S}_{\text {enz-active }}
$$

then,

$$
\begin{aligned}
& \Delta \mathrm{S}_{\text {enz-active }} \\
& =\Delta \mathrm{S}_{\text {torsions }}+\Delta \mathrm{S}_{\text {rotations }}+\Delta \mathrm{S}_{\text {vibrations }}+\Delta \mathrm{S}_{\text {stretching }} \\
& +\Delta \mathrm{S}_{\mathrm{H} \text {-bond }}+\Delta \mathrm{S}_{\text {van der Waal }} \Delta \mathrm{S}_{\text {Coloumb }}+a \text {. }
\end{aligned}
$$

However

$$
\Delta \mathrm{S}_{\text {enz-active }}=\Delta \mathrm{S}_{\mathrm{enz}-\mathrm{relax}}+a+\delta,
$$

where $\delta$ is the additional activation entropy which acts as an energetic expense for the enzyme conformation change from passive to active to occur.

In the example of the voltage-gated ion pump, the $\delta$ factor is given by the sensor domains' activation of the pump and proves to be continuously different from an adjacent pump who works at a slightly different ion concentration. So,

$$
\Delta \mathrm{S}_{\text {enz-active }} \neq \Delta \mathrm{S}_{\text {enz-activel }} \neq \Delta \mathrm{S}_{\text {enz-active2 }}
$$

where the entropies are, respectively, of enzyme pump 1, enzyme pump 2, and enzyme pump 3. The differences in entropy drive the different points of activation of the various sensor domains yielding a continuous pumping of ions catalyzed by nonsynchronized enzyme activations. This itself maintains the variation of ion concentration in the cell and thereby, a continuous active/passive system drives and is driven by the ion concentration fluctuations.

\section{Delineating Continuous Activity between the Nanolayers}

As depicted in the nanocell in Figure 1, the start concentration of ions within a nanolayer of for instance $\mathrm{KCl}$ is given by

$$
[\mathrm{KCl}]_{0} \Longleftrightarrow\left[\mathrm{K}^{+}\right]_{0}+\left[\mathrm{Cl}^{-}\right]_{0},
$$

with its dissociation constant $k_{\text {dis }}$

As an engineering act, each nanolayer (see Figure 1) is made to contain a different start concentration $\left[\mathrm{K}^{+}\right]_{0}$ with increasing value towards the periphery. So

$$
\left[\mathrm{K}^{+}\right]_{0 \text { nanolayer 2 }}>\left[\mathrm{K}^{+}\right]_{0 \text { nanolayer } 1} \text {. }
$$

Viewing the role of the sensor domain as an activator, we define its role in the system as $\beta$.

(9) $\beta$ is the sensor factor, relating the potential of activating an enzyme in a given timeframe and is therefore defined as change in $\Delta S_{\text {enz }},\left(\Delta \Delta S_{\text {enz }}\right)$ per time unit: for instance $\mathrm{kJ} / \mathrm{fs}$ (femtosecond).

Having in mind that the change in ion concentration is the trigger of the pumping function, then the following statements can be deduced when the system moves forward to reach equilibrium:

$$
\Delta\left[\mathrm{K}^{+}\right]_{\text {nanolayer } 1} \cong \Delta\left[\mathrm{K}^{+}\right]_{\text {nanolayer } 2},
$$

and $\beta$ remains constant (including non-additive contributions), given the premises in (8) and that all enzymes are identical in structure and function.

Therefore,

$$
\Delta \Delta\left[\mathrm{K}^{+}\right]_{\text {nanolayers }}>0,
$$

remaining a positive value given (8) and given that the pumps are active and the force of Le Chatelier's principle is equal to the force exerted by the enzymes.

The entropic contributions within a layer are defined by the change in ion concentrations:

$$
\Delta \Delta \mathrm{S}=\Delta \mathrm{S}_{0}-\Delta \mathrm{S}_{1}
$$

with respect to concentration of ions at time 0 and at time 1 .

Therefore,

$$
\sum \Delta \Delta \mathrm{S}_{\text {nanolayer } 1}=\Delta \Delta \mathrm{S}_{\mathrm{enz1}}+\Delta \Delta \mathrm{S}_{\mathrm{enz2}}+\cdots+\Delta \Delta \mathrm{S}_{\mathrm{enz} n} \text {, }
$$


where each enzyme integrated in the membrane is included in the sum.

And so

$$
\sum \Delta \Delta \mathrm{S}_{\text {nanolayer } 2} \neq \sum \Delta \Delta \mathrm{S}_{\text {nanolayer } 1},
$$

because of (11).

Given that $\beta$ in (9) reaches a constant value, this yields that also $\Delta \Delta \mathrm{S}$ within a nanolayer remains constant. As $\Delta \Delta \mathrm{S}$ remains constant, also $\Delta \Delta\left[\mathrm{K}^{+}\right]_{\text {nanolayers }}$ remains constant, as described in (11).

When $\Delta \Delta\left[\mathrm{K}^{+}\right]_{\text {nanolayers }}$ remains constant at its positive value, then

$$
\sum \Delta \Delta \mathrm{S}_{\text {nanolayer } 2}-\sum \Delta \Delta \mathrm{S}_{\text {nanolayer } 1}<0,
$$

because of (11).

And increasing the number of layers, $n$ :

$$
\lim _{n \rightarrow \infty} \Delta \Delta \mathrm{S}_{\text {system }}<0 \text {. }
$$

And because

$$
\begin{aligned}
& \sum \Delta \Delta \mathrm{S}_{\text {nanolayer 2 }}-\sum \Delta \Delta \mathrm{S}_{\text {nanolayer 1 }}<0, \\
& \sum \Delta \Delta \mathrm{S}_{\text {nanolayer 2 }}-\sum \Delta \Delta \mathrm{S}_{\text {nanolayer 1 }} \\
& \quad \in \sum \Delta \Delta \mathrm{S}_{\text {nanolayer 3 }}-\sum \Delta \Delta \mathrm{S}_{\text {nanolayer 2 }},
\end{aligned}
$$

then

$$
\Delta \Delta S_{\text {system }}<0 .
$$

This is sustained by the fact that $\beta$ reaches a constant value, and $\Delta \Delta\left[\mathrm{K}^{+}\right]_{\text {nanolayers }}$ never reaches the value of 0 , assuming that the system remains intact and that Le Chatelier's principle is counterforced by a suitable number of ionpumps which are working normally.

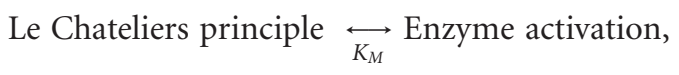

where Le Chatelier's principle as a force attempts to equilibrate the differing concentrations between the layers, while the enzymes in two opposite directions attempt to keep the concentrations as they are at start according to their voltagesensing properties [15]. The equilibrium constant $K_{M}$ as noted here is defining the cell's overall catalytic activity in generating impulses on the containing nanomaterials with phototrophic, magnetotactic, or morphomutating properties.

\section{A Different View of the Energy Contributions}

In order to view the system in accordance with its reining forces, the forces that move the ions according with Le Chatelier's Principle and the forces exerted by the ion pumps are described, respectively, as

$$
\overrightarrow{\mathrm{LC}} \text { and } \overrightarrow{\mathrm{IP}} \text {, }
$$

and if a nanoenergy cell is engineered with a suitable arrangement of enzymes to satisfy that the overall impulses exerted by the forces are equal to Le Chatelier's force over an infinite period of time such that the following criteria is satisfied:

$$
\int_{0}^{\infty} \overrightarrow{\mathrm{LC}} \equiv \int_{0}^{\infty} \overrightarrow{\mathrm{IP}}
$$

Then, in a given timeframe $t$ between 0 and infinitely many seconds, with an intact system, the enzyme population being chosen to exert an equivalent force to Le Chatelier's Principle, the systems' ion concentration homogeneity, delineated by the symbol $\Omega$, will attempt to reach zero however because of the non-additive contemplations shown in the next paragraph, does not:

$$
\lim _{t \rightarrow \infty} \Omega>0,
$$

where (22) shows a presence of ionic inhomogeneity in the system if the terms of (21) are satisfied. The in-homogeneity builds the foundation for driving the ion sensors, and thereby driving the fuel cell.

\section{Non-additive Contributions}

The non-additive contributions are assumed here to be exerted on the enzymes which are localized in different layers that separate different ionic concentrations (chambers). The non-additive contributions describe that the enzymes located in the $\mathrm{n}$-th layer are not equally contributing to ionconcentration changes as the enzymes located in another layer on the threshold of different ionic concentrations. They key to enzyme activity is ionic inhomogeneity, $\Omega$ (22). Therefore, the difference in the contribution of the enzymes in different layers to the factor $\Omega$, yields the nonconventional assumption on that the ionic concentration will never reach equilibrium if the cell is composed of enough layers.

In order to outline the contribution of non-additive effects, the work supplied by Matsuura and colleagues [27] is used to model the non-additive contributions to the assumptions of continuity outlined in the previous paragraphs. The work by Matsuura and colleagues [27] described the non-additive contributions to the properties of a population of enzymes (of wild types and mutants). Here in the nanoenergy cell theory, the enzymes working under ideal ionic concentrations and an appropriate electrostatic potential as calculated by Alabi et al. 2007 [15] are viewed as wild types, while enzymes working under nonideal electrostatic conditions are viewed as mutants.

As Matsuura and colleagues [27] outlined the difference between mutants and wild types is described as:

$$
z=x-x_{L}
$$

Simplifying the number of influences by the factor $\Omega$ on the enzymes activity by Le Chatelier's principle to 
the number $m$, and these influences occur in an arbitrary order, one by one, the difference $z$ can be expressed as

$$
z=\sum_{i=1}^{m} z_{i}+\left(\frac{1}{2}\right) \sum_{i=1}^{m} \sum_{\substack{j=1 \\ j \neq 1}}^{m},
$$

where $z_{i}$ is the change in enzyme kinetics caused by Le Chatelier's Principle at the $i$ th enzyme. Following the rationale given by Matsuura and colleagues:

$$
z_{i}=-b\left(z_{i}\right)
$$

where $b$ is the constant showing the degree of nonadditivity. In order to express the essential effects of nonadditivity on statistical average and variance of the pumping impulses exerted by the enzymes, (8) and (9) are used to express the average of $z$ in the population of enzymes in layer 1 of the nanofuel cell, $E\left(z_{1}\right)$

$$
E\left(z_{1}\right)=E\left(\sum_{i=1}^{m} z_{i}\right)-\left(\frac{b}{2}\right) E\left[\sum_{i=1}^{m} \sum_{\substack{j=1 \\ j \neq 1}}^{m}\left(z_{i}\right)\right] .
$$

The nanocell's nonadditivity is described therefore on the two sets of enzymes: those working at ideal ionic concentrations and those working at less ideal ionic concentrations, which are both affected by Le Chaterlier's principle. This, following the work by Matsuura et al. [27], yields the simplified reasoning of the value of the modeled nonadditivity:

$$
\mathrm{NA}_{\text {hyp }}=E\left(z_{1}+z_{M}\right) \text {. }
$$

Given that the number of enzymes working under ideal conditions, and those working under less ideal conditions is in constant change, nonadditivity effects on the system is itself in constant change.

Therefore, if (21) is reachable in an engineered achievement, the drive force for continuity in the cell can be presented by the following simple mathematical assumption:

\section{Universal generation of nonadditivity}

$$
\overleftrightarrow{K_{M}} \text { Universal need to reach equilibrium, }
$$

where the universal need to reach equilibrium (here in this case, Le Chatelier's principle-is used as a force to drive generation of energy) and is counteracted by nonadditive contributions within the contained system. The product of this assumed continuous attempt of balance denoted by $K_{M}$ in (28) is ion fluctuations, and the ion fluctuations are used to generate energy in the interaction with the nanocomposite. In other systems other products of the balance described in (28) can be depolarizations, reorientations, and other nanosized phenomena that can, if applying hypersensitive work mechanisms such as enzymes, aid in exploiting the forces from the quantum mechanical level better than nonbiological units and mesostructures. Such nanosized units can be parallel

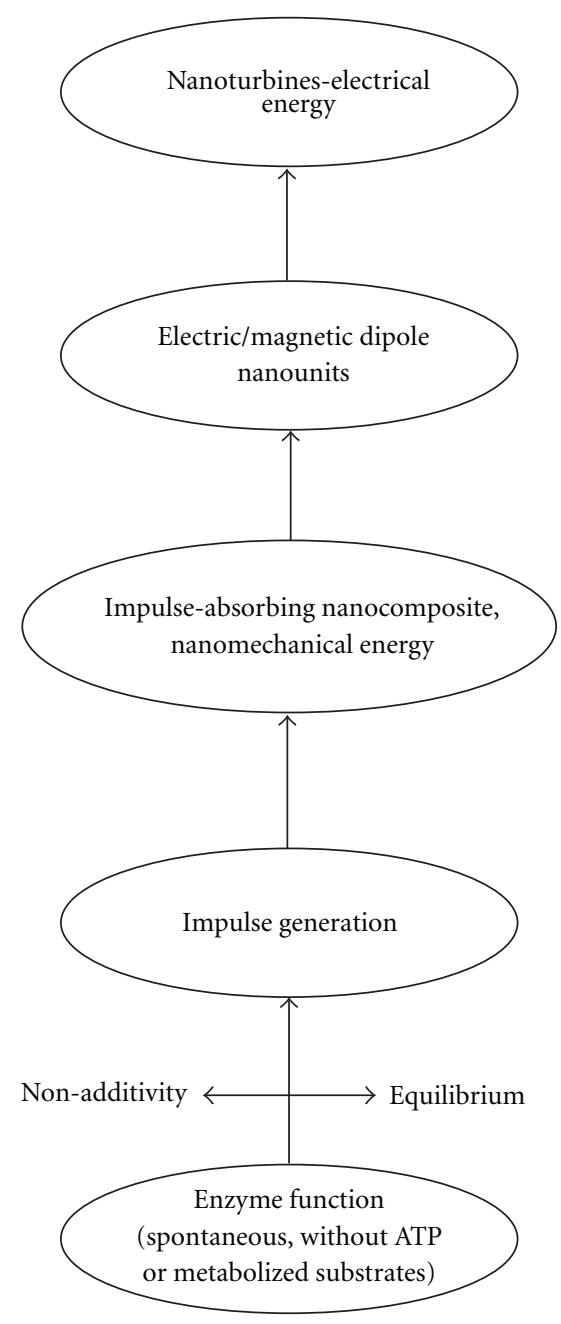

FIGURE 2: The process of extending enzyme functions into energy sources. The diagram shows where the function of the enzyme can be of particular value if it is repeatedly exerted through, without metabolic substrates, with non-additive influences on the system to induce continuity as an opposing force to equilibrium. The impulse generated from the enzyme can be translated into mechanical, morphologic or phototrophic nanosized signals. The flux of particles, water or ions which can in non-additive conditions drive the enzymes to perturbate their functionality continuously. The fluctuations in the system generate the impulse which affects nanocomposite properties which in turn are exploited to affect rotation and exchange of electrons in nanoturbines via nanomechanical switches/components.

connected to a main source to generate sums of enzyme energies.

This theoretical approach to the application of biomolecules in nanoenergetics and nanotechnology is intended to lead the field of nanotechnology researchers into ideas that lift the energy research from its present state where fuel and emissions are an inseparable couple. The diagram shown below (Figure 2) can be a general direction in understanding of the architecting of such inventions. 


\section{Conclusions}

We are here describing the drive of energy through the existence of ionic gradients in a prefabricated manner in a nanotechnological unit. The differing ionic gradients between different compartments stimulate two main forces: the ion pump's forces through the sensors activation and Le Chatelier's Principle forces by moving ions from high to low concentration. The sensor domain requires a difference in ion concentration to be activated, which is seen as the "no cost" of energy to carry out the pumping function, which affects a surrounding nanocomposite with energy transforming qualities. The protein function itself is spontaneous such as with folding pathways are, and is hereby triggered by ion fluctuations. The Principle of Le Chatelier counteracts the pumps' attempt to pump the ions by having inserted in the various layers various concentration of ions from the fabrication process. Once the fabrication process of the layers with high/low ion concentrations is artificially made and the pumps meanwhile exert their pumping function, a potential attempt to reach equilibrium between Le Chatelier's Principle and enzymatic function is hypothesized to continuously be driven by fluctuations as long as the pumps exert a force equal to Le Chatelier's principle (depending on the size of the cell, subdivision of enzyme pumps in opposite directions, etc.). There is however an expected contribution to continuity by non-additive contributions, given that the differences in enzyme activity are dependent on the electrostatic potential. The non-additive contributions to ion fluctuations (and thereby to energy source itself) are expected to increase with an increasing number of layers and ionic chambers with appropriate numbers of enzymes incorporated. This has the effect of triggering the sensitivity of the sensor domains on a continuous basis given that the enzymes work at different rates according to different electrostatic potentials and nonhomogenous distribution of ions in the layers. Simultaneously, $\Delta \mathrm{U}$ is constant being in the closed system.

\section{Acknowledgment}

The author would like to thank Dr. Otto Andersen and Dr. Terje Aaberge from the Western Norway Research Institute for inspiration to write this paper.

\section{References}

[1] L. Cabral, "The California energy crisis," Japan and the World Economy, vol. 14, no. 3, pp. 335-339, 2002.

[2] L. P. Rosa and L. L. B. Lomardo, "The Brazilian energy crisis and a study to support building efficiency legislation," Energy and Buildings, vol. 36, no. 2, pp. 89-95, 2004.

[3] J. S. Ramos and H. M. Ramos, "Sustainable application of renewable sources in water pumping systems: optimized energy system configuration," Energy Policy, vol. 37, no. 2, pp. 633-643, 2009.

[4] A. Skoglund, M. Leijon, A. Rehn, M. Lindahl, and R. Waters, "On the physics of power, energy and economics of renewable electric energy sources-part II," Renewable Energy, vol. 35, no. 8, pp. 1735-1740, 2010.
[5] G. Tegart, "Energy and nanotechnologies: priority areas for Australia's future," Technological Forecasting and Social Change, vol. 76, no. 9, pp. 1240-1246, 2009.

[6] E. Serrano, G. Rus, and J. García-Martínez, "Nanotechnology for sustainable energy," Renewable and Sustainable Energy Reviews, vol. 13, no. 9, pp. 2373-2384, 2009.

[7] M. Zäch, C. Hägglund, D. Chakarov, and B. Kasemo, "Nanoscience and nanotechnology for advanced energy systems," Current Opinion in Solid State and Materials Science, vol. 10, no. 3-4, pp. 132-143, 2006.

[8] S. Mann, "Self-assembly and transformation of hybrid nanoobjects and nanostructures under equilibrium and nonequilibrium conditions," Nature Materials, vol. 8, no. 10, pp. 781-792, 2009.

[9] R. Lipowsky, J. Beeg, R. Dimova, S. Klumpp, and M. J. I. Müller, "Cooperative behavior of molecular motors: cargo transport and traffic phenomena," Physica E, vol. 42, no. 3, pp. 649-661, 2010.

[10] S. C. J. Huang, A. B. Artyukhin, N. Misra et al., "Carbon nanotube transistor controlled by a biological ion pump gate," Nano Letters, vol. 10, no. 5, pp. 1812-1816, 2010.

[11] Y. Li-Smerin, D. H. Hackos, and K. J. Swartz, "A localized interaction surface for voltage-sensing domains on the pore domain of a K+ channel," Neuron, vol. 25, no. 2, pp. 411-423, 2000.

[12] Y. Li-Smerin, D. H. Hackos, and K. J. Swartz, " $\alpha$-Helical structural elements within the voltage-sensing domains of a $\mathrm{K}$ channel," Journal of General Physiology, vol. 115, no. 1, pp. 3349, 2000.

[13] Y. Jiang, A. Lee, J. Chen et al., "X-ray structure of a voltagedependent K+ channel," Nature, vol. 423, no. 6935, pp. 33-41, 2003.

[14] Y. Kubo, T. J. Baldwin, Y. N. Jan, and L. Y. Jan, "Primary structure and functional expression of a mouse inward rectifier potassium channel," Nature, vol. 362, no. 6416, pp. 127-133, 1993.

[15] A. A. Alabi, M. I. Bahamonde, H. J. Jung, J. I. Kim, and K. J. Swartz, "Portability of paddle motif function and pharmacology in voltage sensors," Nature, vol. 450, no. 7168, pp. 370-375, 2007.

[16] A. M. Seddon, H. M. Patel, S. L. Burkett, and S. Mann, "Chiral templating of silica-lipid lamellar mesophase with helical tubular architecture," Angewandte Chemie, vol. 41, no. 16, pp. 2988-2991, 2002.

[17] S. B. Brichkin, M. A. Osipova, T. M. Nikolaeva, and V. F. Razumov, "The hybrid nanosystem nanosized silver halide grain-dye in AOT reverse micelles," High Energy Chemistry, vol. 39, no. 6, pp. 386-391, 2005.

[18] E. D. Spoerke, G. D. Bachand, J. Liu, D. Sasaki, and B. C. Bunker, "Directing the polar organization of microtubules," Langmuir, vol. 24, no. 14, pp. 7039-7043, 2008.

[19] T. M. Chen, A. C. Graham, M. Pepper et al., "Direct observation of nonequilibrium spin population in quasi-onedimensional nanostructures," Nano Letters, vol. 10, no. 7, pp. 2330-2334, 2010.

[20] H. Liu, J. J. Schmidt, G. D. Bachand et al., "Control of a biomolecular motor-powered nanodevice with an engineered chemical switch," Nature Materials, vol. 1, no. 3, pp. 173-178, 2002.

[21] H. Liu, E. D. Spoerke, M. Bachand, S. J. Koch, B. C. Bunker, and G. D. Bachand, "Biomolecular motor-powered self-assembly of dissipative nanocomposite rings," Advanced Materials, vol. 20, no. 23, pp. 4476-4481, 2008. 
[22] S. Mann, "Self-assembly and transformation of hybrid nanoobjects and nanostructures under equilibrium and nonequilibrium conditions," Nature Materials, vol. 8, no. 10, pp. 781-792, 2009.

[23] E. J. Parra, G. A. Crespo, J. Riu, A. Ruiz, and F. X. Rius, "Ionselective electrodes using multi-walled carbon nanotubes as ion-to-electron transducers for the detection of perchlorate," Analyst, vol. 134, no. 9, pp. 1905-1910, 2009.

[24] M. L.. Górzny, A. S. Walton, M. Wnęk, P. G. Stockley, and S. D. Evans, "Four-probe electrical characterization of Pt-coated TMV-based nanostructures," Nanotechnology, vol. 19, no. 16, Article ID 165704, 2008.

[25] Z. Tang, N. A. Kotov, and M. Giersig, "Spontaneous organization of single CdTe nanoparticles into luminescent nanowires," Science, vol. 297, no. 5579, pp. 237-240, 2002.

[26] T. Tlusty and S. A. Safran, "Defect-induced phase separation in dipolar fluids," Science, vol. 290, no. 5495, pp. 1328-1331, 2000.

[27] T. Matsuura, T. Yomo, S. Trakulnaleamsai, Y. Ohashi, K. Yamamoto, and I. Urabe, "Nonadditivity of mutational effects on the properties of catalase I and its application to efficient directed evolution," Protein Engineering, vol. 11, no. 9, pp. 789-795, 1998. 

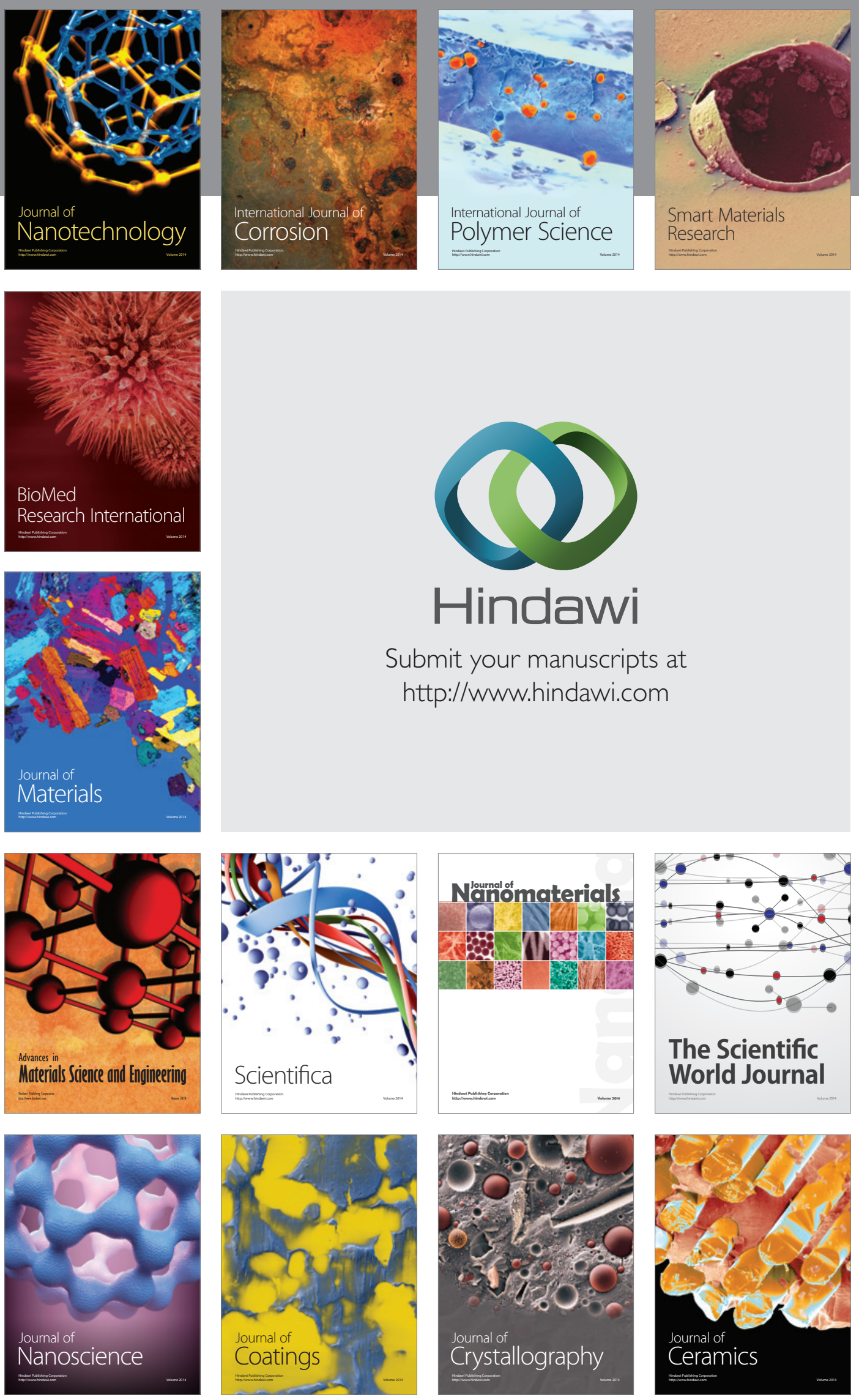

The Scientific World Journal

Submit your manuscripts at

http://www.hindawi.com

\section{World Journal}

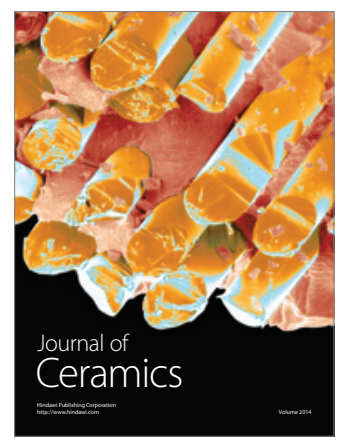

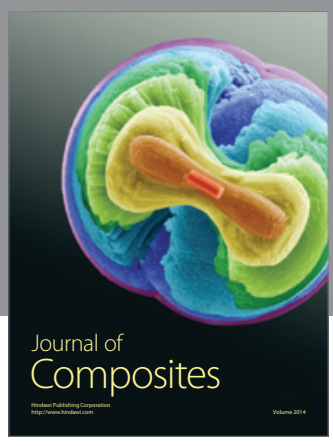
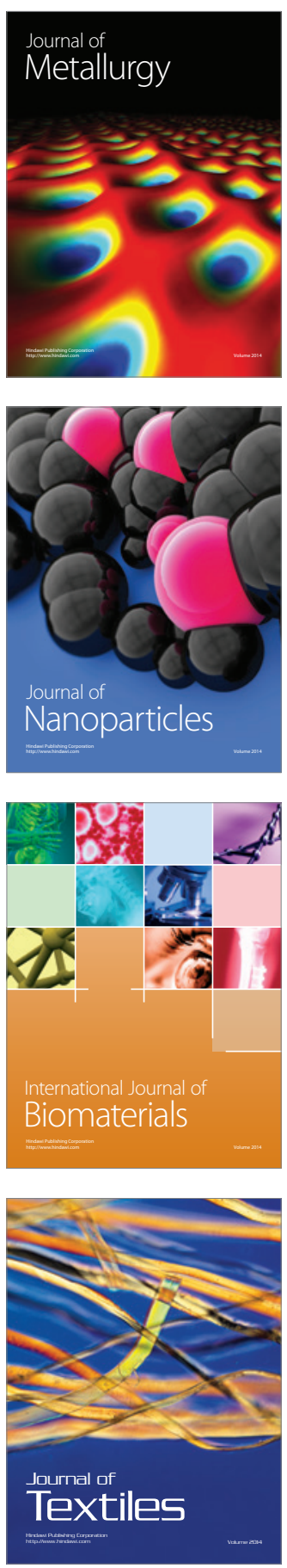